Article

\title{
Economic Microgrid Planning Algorithm with Electric Vehicle Charging Demands
}

\author{
Sung-Guk Yoon * and Seok-Gu Kang \\ Department of Electrical Engineering, Soongsil University, Seoul 06978, Korea; vtz@naver.com \\ * Correspondence: sgyoon@ssu.ac.kr; Tel.: +82-2-828-7050 \\ Academic Editor: Rui Xiong \\ Received: 4 September 2017; Accepted: 18 September 2017; Published: 25 September 2017
}

\begin{abstract}
Two of the most important technologies for future power systems to reduce greenhouse gas are electric vehicles (EVs) and renewable generation. When EVs become more common, the overall demand of electricity will significantly increase because EVs consume a large amount of electricity. Also, a daily load curve with EVs heavily depends on how much electricity EVs consume and when electricity is consumed. The microgrid is an important technology to promote renewable generation, and the increased demand and changed load curve should be considered in the microgrid planning stage to install robust and economical microgrids. In this paper, we propose an algorithm for microgrid planning with EV charging demand to find the most economical configuration through which to maximally utilize renewable generation. The algorithm uses a renewable generation-following EV charging scheme and HOMER. Through simulations, it is shown that the microgrid constructed by the proposed algorithm reduces the investment cost and $\mathrm{CO}_{2}$ emission.
\end{abstract}

Keywords: electric vehicle; EV charging scheduling; microgrid planning; HOMER

\section{Introduction}

Sustainable development is one of the key engineering challenges for future society. To this end, 195 countries agreed in December 2015 to reduce greenhouse gas through the Paris agreement [1]. According to the agreement, Korea must reduce greenhouse emissions $37 \%$ by 2030 compared to that of business-as-usual (BAU). From the perspective of the power system, two of the most vital technologies to reduce greenhouse gas are electric vehicles (EVs) and renewable generation. Note that in this paper, an EV indicates any battery-type plug-in electric vehicle such as a plug-in hybrid electric vehicle (PHEV) or plug-in electric vehicle (PEV).

EVs are receiving attention as a promising alternative to the fossil fuel-based vehicles that are one of the major causes of the greenhouse effect [2]. However, since EVs consume a very high amount of electricity during the charging period, the power system will be exposed to considerable stress when EVs become widespread [3]. It has been shown that simple electricity price rates such as time-of-use (TOU) pricing induce a new rebound peak in the off-peak hours because most EVs are mainly charged during that period [4].

Many studies have been conducted on reducing the stress on the grid by distributing the EV charging demand, i.e., the EV charging scheduling issue [4-11]. Distributing methods for EV charging demands can be classified into centralized [5-8] or decentralized [9-11] schemes. In [5], a real-time EV charging scheme has been proposed to improve voltage profile through centralized charging control. In addition, the authors of [6] have proposed various objective functions such as total feeder losses, total cost of energy, and total cost of PEV charging to protect the distribution grid. To reduce computational complexity, a scalable EV charging scheduling scheme has been proposed through Model Predictive Control (MPC) by using statistical information [7]. In [8], a semi-centralized scheme 
has been proposed in which, on behalf of each EV owner, a fleet operator interacts with a distribution system operator to determine the hourly electricity price. Conversely, in [9-11], a decentralized scheme has been proposed. The EV scheduling was modeled as a Stackelberg game, in which the players are an electricity retailer and EV customers [9]. In [10], an optimal EV charging scheme has been proposed to fill the valleys of electric load curve. Last, with an assumption of predictable EV user's behavior, an optimal EV scheduling has been proposed with discrete EV charging levels [11].

The microgrid is a vital technology to promote renewable generation to the power system. It is defined as a group of interconnected loads and distributed energy resources with clearly defined electrical boundaries. The two major advantages of the microgrid are: (i) a number of distributed generations can be adopted in the grid through the microgrid [12]; and (ii) the microgrid improves the grid resilience against disasters [13].

Since microgrids include fewer households and consume less electricity than a main grid, the adoption of EVs in a microgrid will have a more serious impact. Therefore, EV charging in microgrids should be precisely controlled to ensure their economy and resilience. To this end, recent studies have investigated the EV charging issue in the microgrid [14,15].

The optimal microgrid planning problem has been investigated through simulation tools such as HOMER and WebOpt $[16,17]$, or through numerical analysis by considering uncertainties in the future [18-20]. In [18], an optimal microgrid planning problem has been proposed for island-mode microgrids while a similar optimization problem has been proposed for grid-connected microgrids in [19]. Another optimization problem for microgrid planning has been proposed that minimizes risk rather than cost [20].

EV charging demands will significantly change the load curve in microgrid, so we should consider EV charging scheduling schemes in the microgrid planning stage. To the best of our knowledge, however, none of the studies consider the load curve changes due to the EVs in the microgrid planning stage.

In this paper, the microgrid planning problem with EV charging demands is investigated. Three main contributions of this work are: (i) we derive load curves from several EV charging scheduling schemes; (ii) optimal microgrid configurations for above charging schemes are investigated using HOMER; and (iii) we propose an algorithm for microgrid planning that maximally uses renewable generators. The proposed algorithm iteratively searches between an optimal EV charging schedule and an optimal microgrid configuration. Through simulations, it is shown that the microgrid constructed by the proposed algorithm reduces the investment cost and $\mathrm{CO}_{2}$ emission by controlling the EV charging demands effectively.

The remainder of the paper is organized as follows. We first describe EV charging scheduling schemes in Section 2. Then, the optimal microgrid planning problem by using HOMER is shown in Section 3. Using the renewable generation-following EV charging scheme and HOMER, we propose an algorithm for microgrid planning in Section 4. After evaluating case studies in Section 5, we conclude our paper in Section 6.

\section{EV Charging Scheduling Schemes}

In this section, the EV charging model is described and the four EV charging scheduling schemes are then shown.

\subsection{EV Charging Model}

We consider a set $\mathcal{N}$ of EVs in a microgrid. Each day is divided into $H$ time periods. A period set and period index are denoted as $\mathcal{H}=\{1,2, \cdots, H\}$ and $h$, respectively. Let $x_{i}^{h}$ denote the amount of electricity consumption by customer $i$ at time $h$ to charge the $\mathrm{EV}$ in units of $\mathrm{kW}$. It is assumed that all EVs are charged at home and on campus for the residential microgrid and campus microgrid, respectively. 
The charging requirement of each EV $i$ is a full charging of its battery before departure. This can be modeled as

$$
\begin{array}{ll}
\sum_{h \in \mathcal{T}_{i}} \mu_{c} x_{i}^{h}=E_{i}, & \\
0 \leq x_{i}^{h} \leq \delta_{i}, & \text { if } h \in \mathcal{T}_{\mathcal{T}^{\prime}} \\
x_{i}^{h}=0, & \text { if } h \notin \mathcal{T}_{i} .
\end{array}
$$

where $\mu_{c}, E_{i}$, and $\delta_{i}$ denote the battery charging efficiency, electricity in kWh required for full charging of EV $i$, and the maximum charging rate of EV $i$, respectively. The charging period for EV $i$ starts at $\alpha_{i}$ and ends at $\beta_{i}$. Let $\mathcal{T}_{i}=\left[\alpha_{i}, \beta_{i}\right]$ denote the set of time periods in which EV $i$ is at the place of charging, i.e., at home or on campus.

\subsection{Min Time Charging Scheduling Scheme}

The most naïve charging scheduling scheme is that EVs are charged as soon as possible. That is, when an EV is parked at home or on campus, it is charged with $\delta_{i}$ charging rate until fully charged. In this paper, we call this scheme the "min time charging scheduling scheme" [21]. It is important to note that this scheme does not require interaction between customers and control entities such as the microgrid energy management system (EMS) and fleet operator, i.e., it is an uncontrolled charging scheme.

\subsection{Min Cost Charging Scheduling Scheme}

Currently, utility companies provide special electricity price rates for EV charging demand. In general, they provide TOU rates by dividing one day into peak, partial-peak, and off-peak periods. Table 1 shows two EV charging price rate examples for Pacific Gas and Electric Company (PG\&E) in the US [22] and Korea Electric Power Corporation (KEPCO) in Korea [23] during the summer season. There is another type of electricity pricing rate named real-time pricing (RTP), which announces hourly price rate for the next day. Southern California Edison (SCE) in the US currently offers RTP in which the hourly price is decided from past daily load data and weather data. Note that a graph of RTP and SMP in one day of summer 2014 in Korea in Section 5 shows examples of TOU and RTP price rates offered by KEPCO.

\begin{tabular}{|c|c|c|c|}
\hline Utility Company & Off-Peak Period & Partial-Peak Period & Peak Period \\
\hline \multirow{2}{*}{ PG\&E } & [11 p.m., 7 a.m.] & $\begin{array}{l}\text { [7 a.m., } 2 \text { p.m.] } \\
{[9 \text { p.m. } 10 \text { p.m.] }}\end{array}$ & [2 p.m., 9 p.m.] \\
\hline & $0.099 \$ / \mathrm{kWh}$ & $0.226 \$ / \mathrm{kWh}$ & $0.428 \$ / \mathrm{kWh}$ \\
\hline \multirow[t]{2}{*}{ КЕРСО } & [11 p.m., 9 a.m.] & $\begin{array}{l}\text { [9 a.m., } 10 \text { a.m.], } \\
{[12 \text { p.m., } 1 \text { p.m.], }} \\
{[5 \text { p.m., } 11 \text { p.m.] }}\end{array}$ & $\begin{array}{l}\text { [10 a.m., } 12 \text { p.m.] } \\
\text { [1 p.m., } 5 \text { p.m.] }\end{array}$ \\
\hline & $0.048 \$ / \mathrm{kWh}^{1}$ & $0.121 \$ / \mathrm{kWh}$ & $0.194 \$ / \mathrm{kWh}$ \\
\hline
\end{tabular}

Table 1. Electric Vehicle (EV) Charging Price Rate for Pacific Gas and Electric Company (PG\&E) and Korea Electric Power Corporation (KEPCO).

${ }^{1}$ We use the exchange rate of $\$ 1=1200$ Won.

The second uncontrolled EV charging scheme is the cost minimization charging scheduling scheme, called the "min cost charging scheduling scheme". In this charging scheme, customers decide the amount of electricity for the EV charging in each period to minimize the cost of electricity [24]. Since we consider two price rates, i.e., TOU rate and RTP, they are called "min cost1" and "min cost2", respectively. The optimization problem can be formulated as

$$
\left(\mathrm{U}_{i}\right) \min _{x_{i}^{h}} \sum_{h \in \mathcal{T}_{i}} p^{h} \cdot x_{i}^{h}
$$




$$
\begin{array}{lll}
\text { subject to } & \sum_{h \in \mathcal{T}_{i}} \mu_{c} x_{i}^{h}=E_{i}, & \\
& 0 \leq x_{i}^{h} \leq \delta_{i}, & \text { if } h \in \mathcal{T}_{i}, \\
& x_{i}^{h}=0, & \text { if } h \notin \mathcal{T}_{i} .
\end{array}
$$

where $p^{h}$ is the electricity price rate at time $h$. It is assumed that the electricity price rate of the day is known to all customers.

The control variable for the problem $\left(\mathrm{U}_{i}\right)$ is only related to each customer $i$ and it does not depend on other customers' charging behaviors. Therefore, each customer decides his/her charging scheduling. That is, this scheme does not require real-time interaction between each customer and the control entity. The only information for customer $i$ needed to solve the problem $\left(\mathrm{U}_{i}\right)$ is the electricity price rate of the day.

Generally, the problem $\left(\mathrm{U}_{i}\right)$ can have many solutions. and we choose the one which has the fastest charging end time because it is the most convenient solution for the EV owner.

\subsection{Min Var Charging Scheduling Scheme}

In the microgrid case, another important metric is the peak electricity demand, since the microgrid should be planned to fulfill the peak demand. Therefore, we choose the objective to be minimizing the maximum demand of one-day load. In general, the problem has more than one solution, so we choose the one that minimizes the variance of one-day load. We call this the "min Var charging scheduling scheme".

To this end, the amount of base load, denoted by $x_{0}^{h}$, as well as EV loads should be considered together. We formulate the optimization problem as

$$
\begin{array}{ll} 
& (\mathrm{C}) \min _{x_{i}^{h}, i \in \mathcal{N}} \operatorname{Var}\left(X^{h}\right) \\
\text { subject to } & \sum_{h \in \mathcal{T}_{i}} \mu_{c} x_{i}^{h}=E_{i}, \quad \forall i \in \mathcal{N}, \\
& 0 \leq x_{i}^{h} \leq \delta_{i}, \quad \text { if } h \in \mathcal{T}_{i}, \forall i \in \mathcal{N}, \\
& x_{i}^{h}=0, \quad \text { if } h \notin \mathcal{T}_{i}, \forall i \in \mathcal{N} .
\end{array}
$$

where $X^{h}$ and $\operatorname{Var}\left(X^{h}\right)$ are the total electricity consumption at $h$ and its variance, respectively. They are defined as $X^{h}=x_{0}^{h}+\sum_{i \in \mathcal{N}} x_{i}^{h}$ and $\operatorname{Var}\left(X^{h}\right)=\frac{1}{H} \sum_{h}\left(X^{h}-\mu\right)^{2}$.

The problem $(\mathrm{C})$ is a standard convex optimization problem, so we can get its solution. Detailed procedures and the closed form solution for the problem (C) are presented in Appendix A.

The control variable for this optimization problem is the charging rates for all of the EVs in the microgrid. Therefore, the problem $(\mathrm{C})$ requires a central control entity in order to obtain the optimal solution and to distribute power to all customers.

Note that, in [15], the EV scheduling problem of minimizing the load variance was proposed without showing its closed form solution, while this work derived the closed form solution for (C). Also, the target of [15] is the EV scheduling in the case of one house while the target of this work is microgrid planning.

\subsection{Max Renewable Charging Scheme}

Since a number of renewable generations will be adopted in a microgrid [12], one key goal for charging scheduling in a microgrid is a maximum use of the renewable generations. Mathematically, the objective of this scheduling scheme is to minimize the difference between electric load and the renewable generation output by controlling the EV charging demands. We call this "max renewable charging scheduling scheme". 
Since the power output of renewable generation displays randomness, we use an expected value for the output of a renewable generation. Let $R^{h}$ denote a random variable defined to be the output of renewable generations at time $h$, and recall that the total electricity consumption at $h$ is $X^{h}$, which consists of base load and EV charging load. Then, we formulate the optimization problem as

$$
\begin{aligned}
& \text { (R) } \min _{x_{i}^{h}, i \in \mathcal{N}}\left(X^{h}-E\left(R^{h}\right)\right)^{2} \\
& \text { subject to } \sum_{h \in \mathcal{T}_{i}} \mu_{c} x_{i}^{h}=E_{i}, \quad \forall i \in \mathcal{N} \text {, } \\
& 0 \leq x_{i}^{h} \leq \delta_{i}, \quad \text { if } h \in \mathcal{T}_{i}, \forall i \in \mathcal{N}, \\
& x_{i}^{h}=0, \quad \text { if } h \notin \mathcal{T}_{i}, \forall i \in \mathcal{N} .
\end{aligned}
$$

We assume that the type and capacity of renewable generations in the microgrid are given. Like the problem $(\mathrm{C})$, the problem $(\mathrm{R})$ is a standard convex optimization problem, so we can get the solution. Because of the space limit, we omit the detailed procedure.

\section{Microgrid Planning Problem}

When the EV charging demand is added to the base load, the load curve significantly changes. The optimal microgrid planning problem for the changed load is described in this section. We use HOMER Energy software to obtain the optimal microgrid configurations.

\subsection{HOMER Energy}

HOMER energy software was originally developed by the US National Renewable Energy Laboratory (NREL) and is commercially available [25]. It is a simulation tool used to find the optimal combination of generations for a microgrid. For this software, basic parameters need to be manually set, including electrical load, diesel price, solar and wind resources, generation capacities, system costs for each generation, etc. The term 'optimal' in HOMER means that the obtained solution has the lowest total net present cost (NPC) [26] to build the microgrid. The total NPC is mathematically defined as

$$
\text { Total NPC }=\sum_{t=0}^{M} \frac{C_{a n n}}{(1+r)^{t}}
$$

where $M, r$, and $C_{a n n}$ are the project lifetime, discount rate, and the total annualized cost during the project lifetime, respectively.

Note that, to obtain the optimal microgrid configuration, HOMER uses an exhaustive search algorithm that simply calculates the NPCs of all candidate solutions and chooses the one with the lowest NPC as an optimal solution [27].

\subsection{Microgrid Component Modeling}

We consider the possible energy supply options to be solar photovoltaics (PV), wind turbine, diesel generator, battery, and alternating current (AC)/direct current (DC) converter. Table 2 shows the system cost for each generator obtained in [28] and default value. The system cost comprises capital cost, replacement cost, and operation and maintenance (O\&M) cost.

The load profiles for the targeted areas include the base load and EV charging demand. The base load curves are obtained from [29], which is an electricity consumption measurement report by the Korean government. The load curves for EV charging are obtained as shown in Section 2 for the various charging schemes. Also, the daily noise of $15 \%$ and hourly noise of $20 \%$ are added to the load.

The daily solar radiation data for Seoul, Korea $\left(37^{\circ} 34^{\prime} \mathrm{N}, 126^{\circ} 58^{\prime} \mathrm{E}\right)$ are obtained from the National Aeronautics and Space Administration (NASA) Surface Meteorology and Solar Energy website [30]. The annual average daily solar radiation for Seoul is $4.05 \mathrm{kWh} / \mathrm{m}^{2} /$ day. The maximum and minimum 
values are $5.54 \mathrm{kWh} / \mathrm{m}^{2} /$ day and $2.44 \mathrm{kWh} / \mathrm{m}^{2} /$ day in May and December, respectively. The diesel price is set as $\$ 1.22$ per liter, which is obtained from the diesel price monitoring website [31]. The project life time of 25 years and the annual interest rate of $6.0 \%$ are used.

Table 2. Unit Component Cost for Microgrid Configuration.

\begin{tabular}{cccc}
\hline Generators & Capital Cost (\$) & Replacement Cost (\$) & O\&M Cost (\$/yr) \\
\hline Solar PV $(20 \mathrm{~kW})$ & 60,000 & 60,000 & 200 \\
Wind turbine $(20 \mathrm{~kW})$ & 100,000 & 100,000 & 1000 \\
Diesel $(50 \mathrm{~kW})$ & 25,000 & 25,000 & 13,000 \\
Battery $(20 \mathrm{kWh})$ & 6000 & 6000 & 200 \\
Converter $(20 \mathrm{~kW})$ & 6000 & 6000 & 0 \\
\hline
\end{tabular}

\section{Max Renewable Microgrid Planning Algorithm}

To construct a robust and economical microgrid with EVs, we propose an algorithm for microgrid planning that uses renewable generation as much as possible.

Figure 1 shows the structure of the proposed microgrid planning algorithm. The proposed algorithm iteratively solves between an EV charging scheduling problem and a microgrid planning problem until the solution converges. The EV charging scheduling problem is $(\mathrm{R})$ because the goal of this algorithm is the maximum use of renewables. To solve the first problem, a microgrid configuration is required as input and its output is the load curve in the microgrid. Conversely, the second problem requires the load curve and it finds an optimal microgrid configuration that minimizes the microgrid construction and operation cost. This solution is obtained using HOMER.

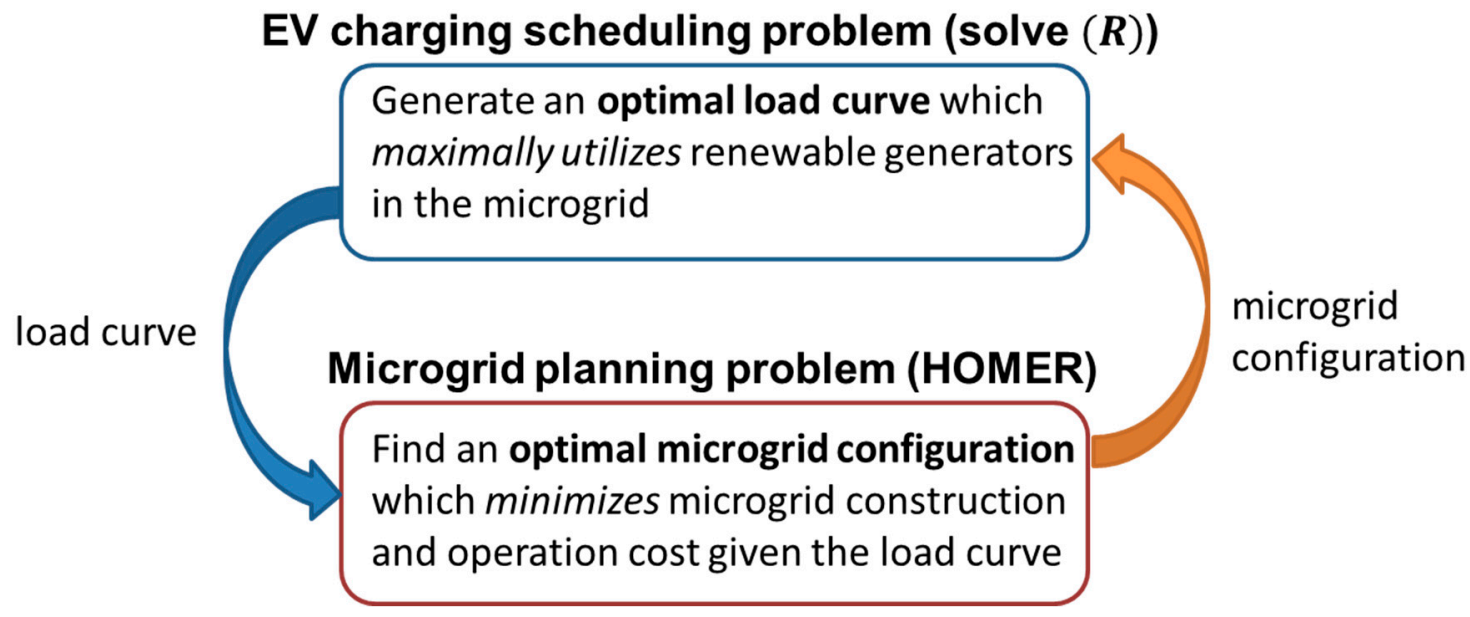

Figure 1. Structure of the proposed microgrid planning algorithm.

The flowchart of the proposed algorithm is shown in Figure 2, and its explanation is as follows.

Step 1: The proposed algorithm begins by setting a default renewable generation type(s) and their capacity with given load curve and diverse EV scheduling schemes.

Step 2: The load curve with EVs using max renewable charging scheme is generated by solving the problem $(\mathrm{R})$ in Section 2.5 .

Step 3: With the updated load curve, the optimal microgrid configuration is investigated using HOMER.

Step 4: We compare the previous and updated configurations, i.e., generation type and capacity, in the microgrid. If they are the same, the proposed algorithm ends. 
Step 4-1: Otherwise, we change the renewable generation types and capacity with the updated ones and the next step is step 2.

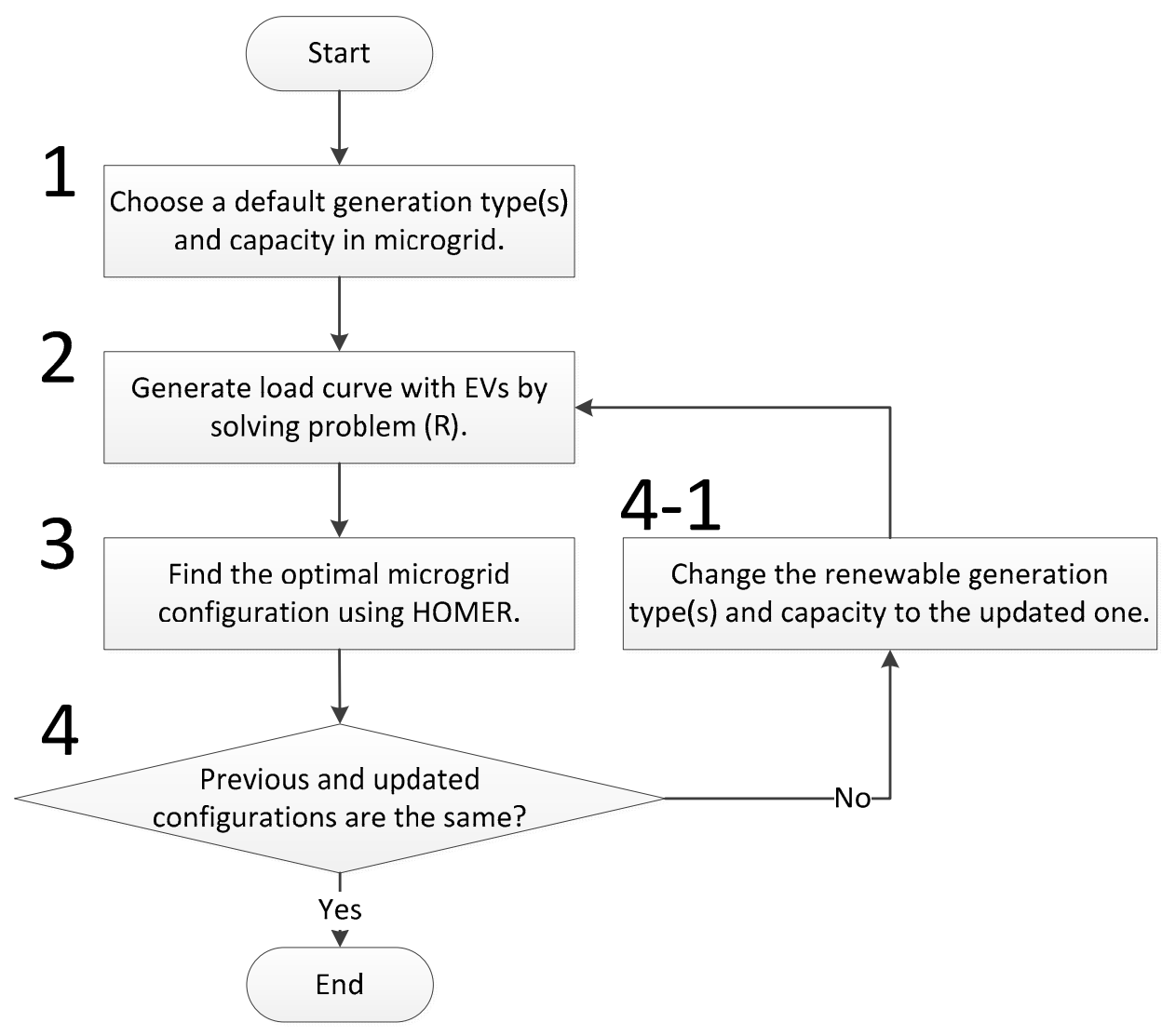

Figure 2. Flowchart for the proposed microgrid planning algorithm.

In the case study, we use a default setting as the min var scheme's microgrid configuration. However, any other microgrid configurations can be used as a default. We confirmed that the same final configuration is obtained even if the microgrid configurations with min time and min cost are used as the default setting.

\section{Case Study}

In this section, two different microgrid cases, i.e., residential microgrid and campus microgrid, are planned at Seoul, Korea. First, the load curves with various EV charging scheduling schemes are presented. Then, optimal microgrid configurations for each load curve are shown.

\subsection{Case Study Settings}

In this case study, one day is divided into $H=24 \mathrm{~h}$ with the time interval of $1 \mathrm{~h}$.

For EV battery modeling, we use the default parameters of Nissan Leaf [32]. That is, the battery capacity is $24 \mathrm{kWh}$ and $\delta_{i}$ for the residential area, i.e., level 2 charging, is $3.3 \mathrm{~kW}$. Average driving efficiency is $5.36 \mathrm{~km} / \mathrm{kWh}$ and $\mu_{c}=0.85$.

For an EV $i$, the EV parameters, i.e., arrival time $\alpha_{i}$, departure time $\beta_{i}$, and battery capacity to charge $E_{i}$, are randomly generated. To obtain the values for these parameters, we use the "2009 National Household Travel Survey" reported by the US Department of Transportation [33]. According to the survey data, the average daily vehicle kilometers of travel is $46.62 \mathrm{~km}$. From the parameters of the Nissan Leaf [32], $10.2 \mathrm{kWh}$ is required to drive $46.62 \mathrm{~km}$. It is then assumed that $E_{i} / \mu_{c}, \alpha_{i}$, and $\beta_{i}$ are 
uniformly distributed in [8 kWh, $12 \mathrm{kWh}$ ], [5 p.m. 9 p.m.], and [5 a.m., 7 a.m.], respectively. Note that the considered arrival and departure time ranges cover more than $90 \%$ of drivers [33].

To use the max renewable charging scheme, $E\left(R^{h}\right)$ in the problem $(\mathrm{R})$ is needed. In this case study, no specific distribution for $R^{h}$ is assumed, but we use real measurement data to get $E\left(R^{h}\right)$. We have the output data of a $30 \mathrm{~kW}$ PV generator in Korea. Then, the output of a PV generator in microgrid is interpolated with the measurement data. For example, if a PV generator has $100 \mathrm{~kW}$ capacity, its output is obtained by multiplying the measurement data by 3.33 .

The TOU-based EV charging price rate of KEPCO is used for the min cost 1 charging scheme. However, the results of this work are not limited to the KEPCO price rate. Any TOU pricing-based EV charging tariff shows a similar result. For the min cost2, i.e., RTP, it is assumed that the system marginal price (SMP) at each hour is the electricity price for the hour. Figure 3 shows the SMP in summer 2014 obtained by Korea Power Exchange and the TOU-based EV charging price rate. Note that the SMP of a day is announced every day, but the TOU price rate is the same.

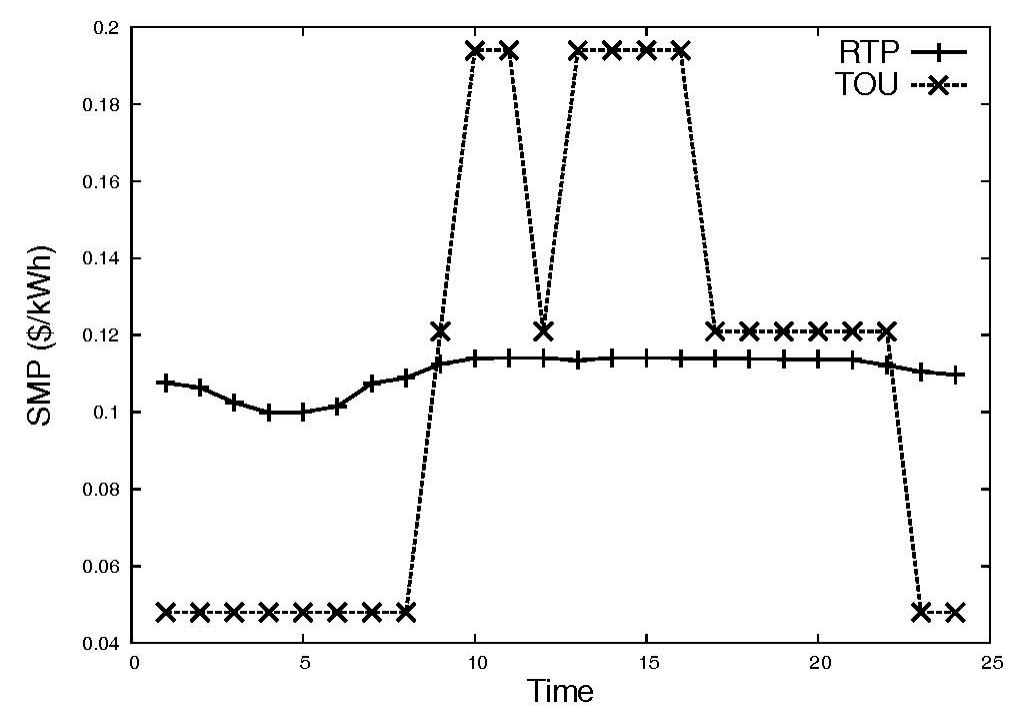

Figure 3. Daily system marginal price (SMP) in summer 2014 in Korea and TOU-based EV charging price rate.

\subsection{Load Curve with EVs}

\subsubsection{Residential Area}

We first consider a microgrid for a residential area with 100 households. A household load curve is obtained from [29]. We use the residential load curve during the summer season, which is shown in Figure 4 as "Base load". The electricity consumption for one day is $9.85 \mathrm{kWh}$ and the peak demand is $0.54 \mathrm{~kW}$ at 10 p.m. With this base load, the EV charging demand is added.

The daily load curve shape changes according to the EV penetration ratio and charging scheduling schemes. Figure 4 shows the load curve for the residential area with the min time, min cost1, min cost2, min Var, and max renewable charging scheduling schemes. We consider $10 \mathrm{EVs}$ and $30 \mathrm{EVs}$ cases, which refer to the EV penetration ratios of $10 \%$ and $30 \%$, respectively. Since we assumed that all EVs return home from 5 p.m. to 9 p.m., and leave home from 5 a.m. to 7 a.m. [33], Figure 4 shows only the period when EVs are parked, i.e., from 5 p.m. to 7 a.m. For the uncontrolled charging scheduling schemes, i.e., min time, min cost1, and min cost2 charging schemes, even if a few EVs (2-3 EVs) exist in the residential area, the peak demand and time are changed. 


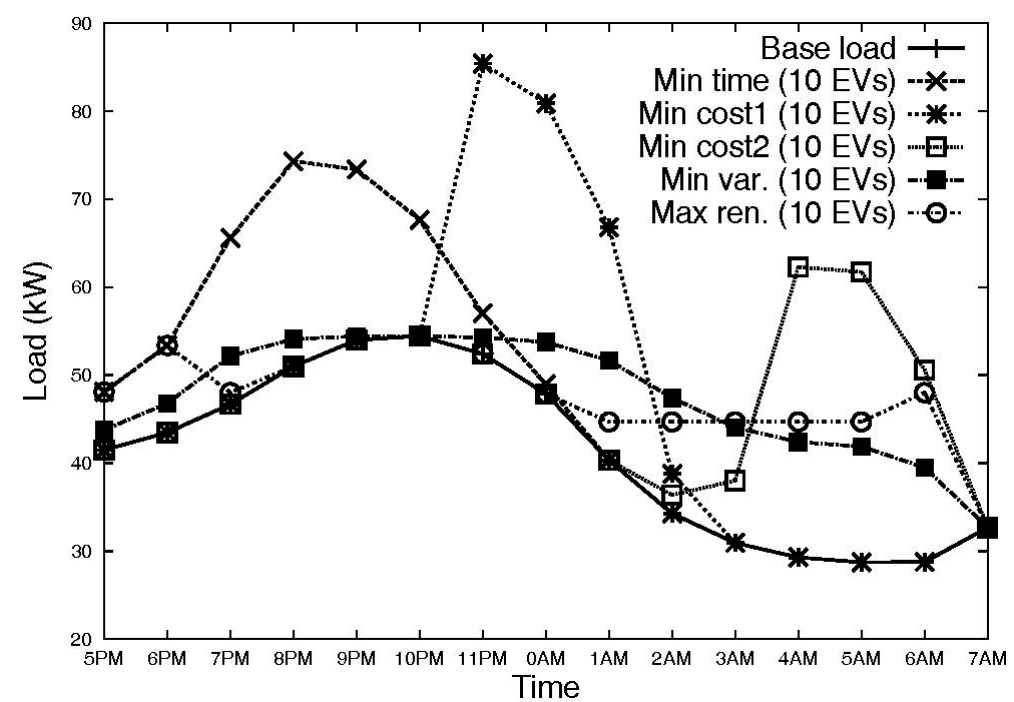

(a)

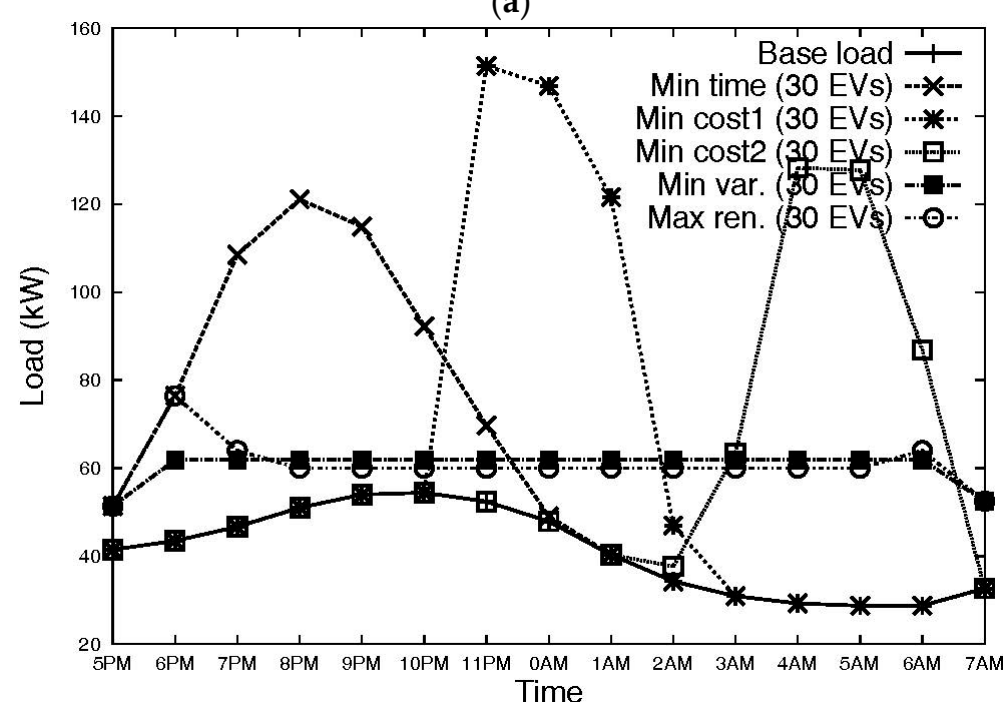

(b)

Figure 4. Load curves from 5 p.m. to 7 a.m. for the residential area. (a) 10 EVs; (b) 30 EVs.

In the case of the min time charging scheme, the charging demands are naturally distributed as the arrival times of EVs are distributed in [5 p.m., 9 p.m.], resulting in less peak demand than that of the min cost schemes. For the min Var charging scheme, the EV charging demand first fills the valley of the off-peak hours. Therefore, the peak demand is not increased to $20 \mathrm{EVs}$. The max renewable charging scheme shows similar load curve to that of min Var charging scheme, but it reveals two small peak demands at 6 p.m. at 6 a.m. since PV generates power at those times.

Note that in this scenario, only PV generation is considered as the renewable generation since wind speed is insufficient to be competitive.

\subsubsection{Campus}

The base load curve for the campus is also obtained from the measurement report in [29]. We use the campus load curve during the summer season, which is shown in Figure 5 as "Base load". The total electricity consumption for one day is $1687 \mathrm{kWh}$ and the peak demand of $115.6 \mathrm{~kW}$ occurred at 2 p.m. 


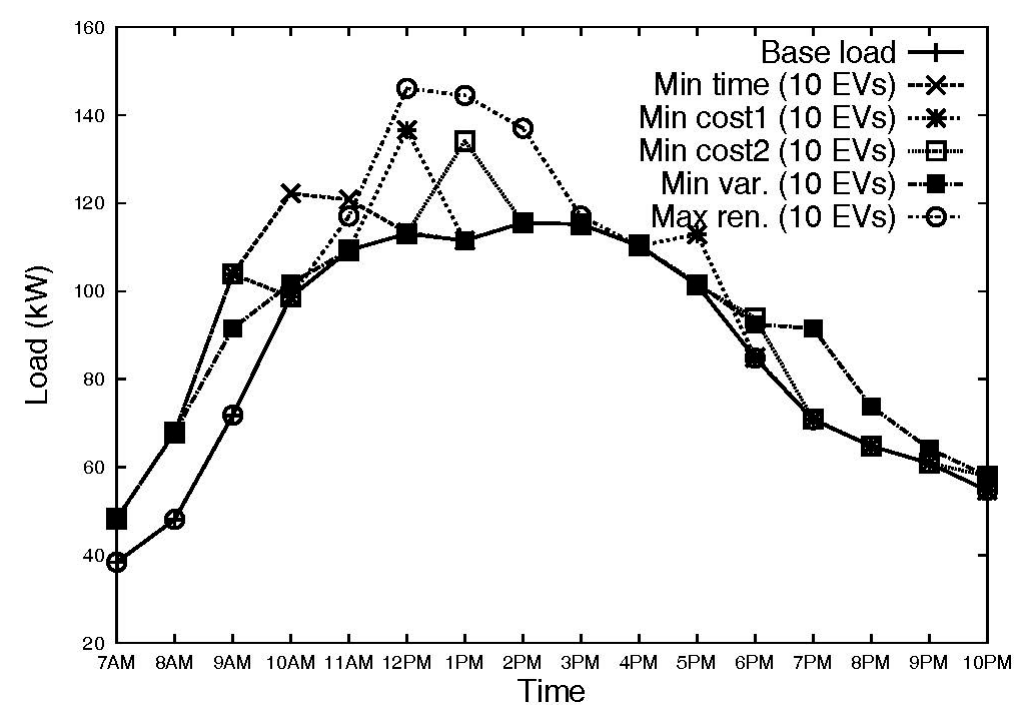

(a)

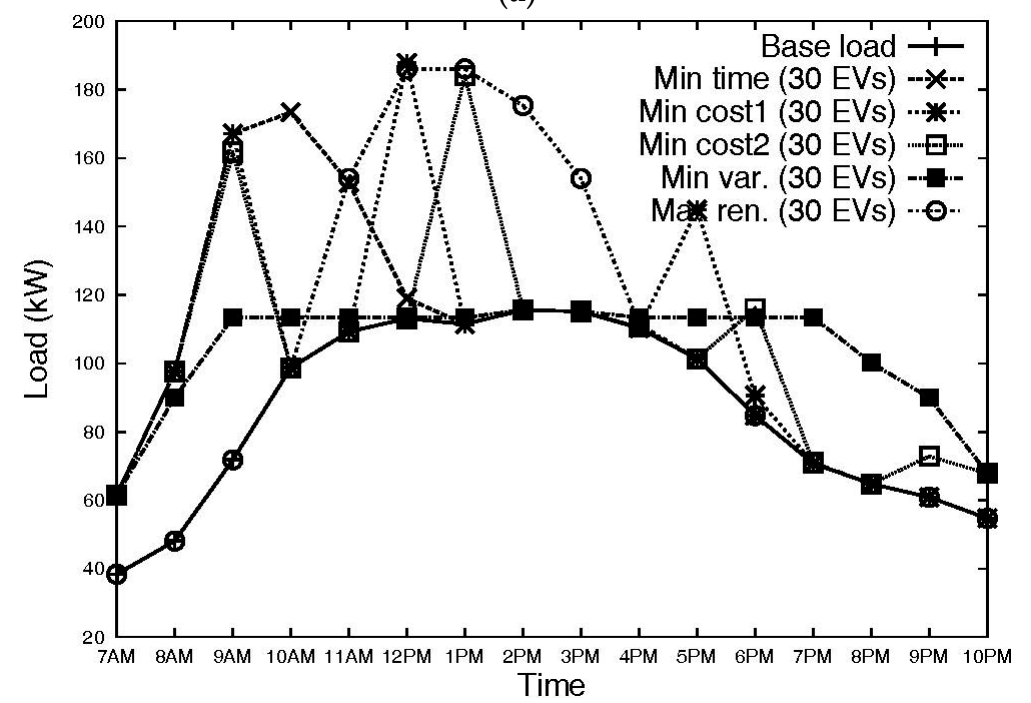

(b)

Figure 5. Load curves from 7 a.m. to 10 p.m. for the campus. (a) 10 EVs; (b) 30 EVs.

The same average daily vehicle kilometers of travel, $\alpha_{i}$, and $\beta_{i}$ are used with an offset of driving time to work. That is, $E_{i} / \mu_{c}, \alpha_{i}$, and $\beta_{i}$ are uniformly distributed in [ $8 \mathrm{kWh}, 12 \mathrm{kWh}$ ], [7 a.m., 9 a.m.], and [5 p.m., 10 p.m.], respectively. In this case, it is assumed that all EVs are charged on the campus. Therefore, Figure 5, which displays the load curve for the campus, shows only the period when EVs are parked at the campus, i.e., from 7 a.m. to 10 p.m.

The min time and min cost charging schemes increase peak demand while the peak demand for the min Var charging scheme is not changed for the $30 \mathrm{EVs}$. The min cost1 charging scheme has three spikes at 9 a.m., 12 p.m., and 5 p.m. because of the KEPCO EV charging price rate. The second spike is the peak demand since the base load at 12 p.m. is the highest among the three spike times. Similar tendency is shown in the min cost 2 charging scheme. The load curve of the max renewable charging scheme shows the same shape as the general PV generation output, and the peak demand of the scheme is the same as those of the min cost charging schemes. 


\subsection{Optimal Microgrid Planning}

With these load curves, the optimal microgrid configurations are obtained using HOMER. In this section, the microgrid operates as the island mode. Section 5.3 discusses the case of the microgrid in grid-connected mode.

\subsubsection{Residential Area}

Figure 6 shows the optimal microgrid configurations for the residential area for various EV charging schemes. The microgrid configurations using the min var charging scheme and the proposed algorithm show considerably similar generation and battery capacities (PV and diesel generations) to the base load since load curves show similar shape as shown in Figure 4. Because they only have $50 \mathrm{~kW}$ diesel generation capacity with $30 \mathrm{EVs}$, more battery capacity is required to make a robust microgrid. On the other hand, the other charging schemes have greater diesel generator capacity to support the peak demand in the 30 EVs scenario.

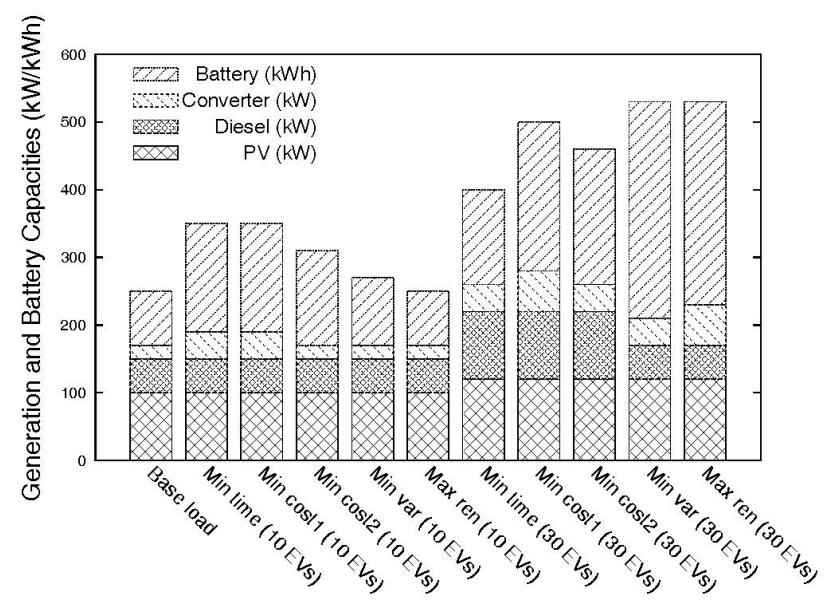

Figure 6. Optimal microgrid configurations for the residential area.

Table 3 shows the investment costs and $\mathrm{CO}_{2}$ emission of the optimal microgrid configurations for each case in detail. The configuration with the min cost 1 charging scheduling scheme and the proposed max renewable microgrid configuration are the most expensive and the least expensive solutions to construct the residential microgrid, respectively. The total NPC differences between scheduling schemes are small with $10 \mathrm{EVs}$, but the gap increases with a number of EVs. The total NPC of the proposed microgrid with $30 \mathrm{EVs}$ saves $10.3 \%$ of the cost compared with that of the min cost 1 scheme with 30 EVs.

Table 3. Investment cost and $\mathrm{CO}_{2}$ emission for the residential microgrid configurations.

\begin{tabular}{ccccc}
\hline & Initial Capital (\$) & Operating Cost $\mathbf{( \$ / y r ) ~}$ & Total NPC (\$) & $\mathbf{C O}_{\mathbf{2}}$ Emission $\mathbf{( k g / \mathbf { y r } )}$ \\
\hline Base load & 355,000 & 122,169 & $1,916,725$ & 206,555 \\
Min time (10 EVs) & 385,000 & 144,970 & $2,238,198$ & 238,495 \\
Min cost1 (10 EVs) & 385,000 & 145,172 & $2,240,789$ & 237,407 \\
Min cost2 (10 EVs) & 373,000 & 132,456 & $2,066,238$ & 223,564 \\
Min var. (10 EVs) & 361,000 & 132,744 & $\mathbf{2 , 0 5 7 , 9 1 2}$ & 226,512 \\
Max ren. (10 EVs) & 355,000 & 133,387 & $2,060,134$ & 229,090 \\
Min time (30 EVs) & 464,000 & 186,050 & $2,842,343$ & 295,585 \\
Min cost1 (30 EVs) & 494,000 & 200,611 & $3,058,487$ & 308,342 \\
Min cost2 (30 EVs) & 482,000 & 181,905 & $2,807,361$ & 285,764 \\
Min var. (30 EVs) & 493,000 & 176,020 & $2,743,129$ & 279,366 \\
Max ren. (30 EVs) & 493,000 & 175,139 & $\mathbf{2 , 7 3 1 , 8 6 7}$ & 279,578 \\
\hline
\end{tabular}


Note that the microgrid with min Var scheme and the proposed microgrid show almost the same result because the PV generation has no impact during the night. In case of the campus microgrid, the two schemes show different results.

\subsubsection{Campus}

Optimal microgrid configurations for the campus are shown in Figure 7. Unlike the residential microgrid, the configuration for the min Var charging scheme is different to that of the proposed microgrid configuration. Due to the nature of maximizing renewable generation, the proposed microgrid requires the highest renewable capacity.

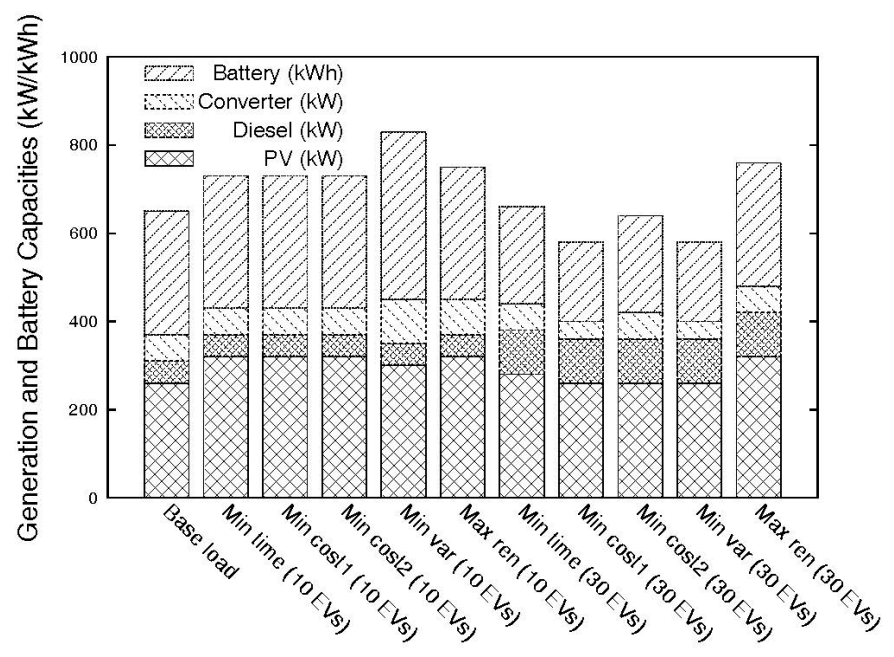

Figure 7. Optimal microgrid configurations for the campus.

Table 4 shows the investment costs and $\mathrm{CO}_{2}$ emission for various EV charging schemes in the campus microgrid. The configuration with the min Var charging scheme and the proposed microgrid configuration show the maximum and minimum total NPC, respectively. Again, the cost savings increase with a number of EVs. Since the load curve of the min Var charging scheme shows a flat shape, PV generation is not a viable solution. Therefore, it mainly depends on diesel generation resulting in the highest cost and $\mathrm{CO}_{2}$ emission. On the other hand, the proposed microgrid maximally utilizes the $\mathrm{PV}$ generation output, so it shows the lowest $\mathrm{CO}_{2}$ emission despite the same diesel generation capacity.

Table 4. Investment cost and $\mathrm{CO}_{2}$ emission for the campus microgrid configurations.

\begin{tabular}{ccccc}
\hline & Initial Capital (\$) & Operating Cost $\mathbf{( \$ / y r ) ~}$ & Total NPC (\$) & $\mathbf{C O}_{\mathbf{2}}$ Emission $\mathbf{( k g / y r )}$ \\
\hline Base load & 907,000 & 164,152 & $3,005,418$ & 267,598 \\
Min time (10 EVs) & $1,093,000$ & 164,866 & $3,200,540$ & 265,982 \\
Min cost1 (10 EVs) & $1,093,000$ & 164,926 & $3,201,313$ & 265,965 \\
Min cost2 (10 EVs) & $1,093,000$ & 165,345 & $3,206,659$ & 266,298 \\
Min var. (10 EVs) & $1,069,000$ & 173,858 & $3,291,483$ & 275,034 \\
Max ren. (10 EVs) & $1,099,000$ & 159,638 & $\mathbf{3 , 1 3 9 , 7 1 4}$ & $\mathbf{2 5 7 , 8 7 6}$ \\
Min time (30 EVs) & 974,000 & 211,326 & $3,675,459$ & 336,167 \\
Min cost1 (30 EVs) & 896,000 & 218,647 & $3,691,036$ & 352,969 \\
Min cost2 (30 EVs) & 914,000 & 217,554 & $3,695,074$ & 349,272 \\
Min var. (30 EVs) & 896,000 & 224,112 & $3,760,900$ & 364,809 \\
Max ren. (30 EVs) & $1,112,000$ & 191,039 & $\mathbf{3 , 5 5 4 , 1 2 5}$ & $\mathbf{2 9 2 , 9 5 5}$ \\
\hline
\end{tabular}

\subsection{Discussion}

The optimal microgrid configuration in the grid-connected mode is also investigated. When the electricity rate of the main grid is set to SMP as shown in Figure 3, the optimal microgrid configurations 
for residential and campus are not cost-effective for installing distributed generators. It is because the electricity price of the main grid is lower than installing distributed generators. Therefore, the daily load curve in the grid-connected mode microgrid is "min cost1" or "min cost2" in Figures 3 and 4 according to the pricing schemes.

As shown in Figures 3 and 4, the current EV pricings (TOU pricing and one day before RTP) fail to distribute EV charging load. Therefore, a more intelligent pricing scheme or EV aggregator, which interacts between retailer and EV customer, is required to distribute the EV charging load [34].

In general, a flat load curve reduces investment and $\mathrm{O} \& \mathrm{M}$ cost in the power system. However, when there are many deferrable loads and renewable generations, the flat daily load curve increases the cost. If the deferrable loads follow the outputs of the renewable generation, we can construct a more economical microgrid.

If the microgrid only has diesel generators, the $\mathrm{CO}_{2}$ emission for residential and campus microgrids are $459,370 \mathrm{~kg} / \mathrm{yr}$ and $692,302 \mathrm{~kg} / \mathrm{yr}$, respectively, in the $30 \mathrm{EVs}$ scenario. That is, the max renewable charging scheme reduces the $\mathrm{CO}_{2}$ emission as $39.1 \%$ and $57.7 \%$ in comparison with those of diesel only microgrid. This result achieves the greenhouse gas reduction target of Korea according to the Paris agreement.

The proposed microgrid planning algorithm theoretically provides a configuration that reveals minimum $\mathrm{CO}_{2}$ emission since it maximally uses renewable generators. In practice, the randomness of renewable generators sometimes causes lower $\mathrm{CO}_{2}$ emissions by other configurations than the proposed algorithm. However, the difference is minimal, i.e., less than $1 \%$, and this case occurrs when the renewable generators make small contributions as shown in the residential microgrid result. The proposed algorithm results in a significant reduction of $\mathrm{CO}_{2}$ emissions when renewable generators contribute significantly like in a campus microgrid.

We also considered the wind turbine option, but our configurations do not include wind turbines because wind speed in Seoul, Korea is too low to be a competitive solution [35].

\section{Conclusions}

To reduce greenhouse gas in the power industry, harmonizing between the two emerging technologies, i.e., microgrid and electric vehicles $(\mathrm{EVs})$ is critical. Therefore, the microgrid planning problem should consider the number of customers in the area who will purchase EVs and how the EV charging loads will be distributed. In this paper, we investigated several uncontrolled and controlled EV charging scheduling schemes: the min time charging scheme, two cost minimization charging schemes, the minimization of variance (min Var) charging scheme, and the max renewable charging scheme. In addition, the optimal microgrid configurations for those charging schemes and different EV penetration ratios were investigated using HOMER. Finally, we proposed a microgrid planning algorithm for a maximum use of renewable generators. The proposed algorithm iteratively solves the EV charging scheduling and microgrid configuration problems to maximally use the renewable generations. Through residential and campus microgrids case studies, the proposed algorithm constructs an economical microgrid with less $\mathrm{CO}_{2}$ emissions. If the installed renewable generation does not produce output power during EV charging, the proposed algorithm works like the min Var charging scheme. Otherwise, it maximizes the use of renewable generations resulting in the lowest investment cost and $\mathrm{CO}_{2}$ emission.

Acknowledgments: This work was supported in part by "Human Resources Program in Energy Technology" of the Korea Institute of Energy Technology Evaluation and Planning (KETEP), granted financial resources by the Ministry of Trade, Industry \& Energy, Republic of Korea (No. 20164010201010), and in part by Korea Electric Power Corporation (Grant number: R17XA05-62).

Author Contributions: Sung-Guk Yoon undertook the major part of this research, and Seok-Gu Kang contributed to the experiment.

Conflicts of Interest: The authors declare no conflict of interest. 


\section{Appendix}

Detailed Procedures to Solve (C)

Here, we derive the solution of the convex optimization problem (C). We begin by solving the problem (C) using a single EV case. The simple case can be formulated as

$$
\begin{array}{cl}
\text { (C1) } \min _{x_{1}^{h}} \frac{1}{H} \sum_{h}\left(X^{h}-\mu\right)^{2} & \\
\text { subject to } & \sum_{h \in \mathcal{T}_{1}} \mu_{c} x_{1}^{h}=E_{i}, \\
& 0 \leq x_{1}^{h} \leq \delta_{i}, \quad \text { if } h \in \mathcal{T}_{1}, \\
x_{1}^{h}=0, & \text { if } h \notin \mathcal{T}_{1} .
\end{array}
$$

From the last constraint, we can reduce the domain of the problem to $h \in \mathcal{T}_{1}$. Also, since the constant term in the objective function has no meaning, we can change it. Then, the problem is changed into

$$
\begin{array}{ll}
\left.\qquad \mathrm{C1}^{\prime}\right) \min _{x_{1}^{h}} \frac{1}{2} \sum_{h}\left(X^{h}-\mu\right)^{2} \\
\text { subject to } \quad \sum_{h \in \mathcal{T}_{1}} \mu_{c} x_{1}^{h}=E_{i}, \\
& 0 \leq x_{1}^{h} \leq \delta_{i}, \quad \forall h .
\end{array}
$$

Its Lagrangian is given by

$$
\begin{aligned}
L\left(\mathbf{x}_{\mathbf{1}}, \boldsymbol{\lambda}_{\mathbf{1}}, \boldsymbol{\lambda}_{\mathbf{2}}, v\right) & =\frac{1}{2} \sum_{h}\left(X^{h}-\mu\right)^{2}+\sum_{h} \lambda_{1 h}\left(x_{1}^{h}-\delta_{1}\right) \\
& -\sum_{h} \lambda_{2 h} x_{1}^{h}+v\left(\sum_{h} x_{1}^{h}-\frac{E_{i}}{\mu_{c}}\right) .
\end{aligned}
$$

where $\mathbf{x}_{1}, \lambda_{1}$, and $\lambda_{2}$ denote the vectors of $x_{1}^{h}, \lambda_{1 h}$, and $\lambda_{2 h}$, respectively, and $\lambda_{1 h}, \lambda_{2 h}$, and $v$ are the Lagrangian multipliers. The optimal solution $\mathbf{x}_{1}^{*}$ for the problem (C1') should satisfy the following KKT conditions [36]

$$
\begin{gathered}
\sum_{h} x_{1}^{h *}-\frac{E_{1}}{\mu_{c}}=0 \\
0 \leq x_{1}^{h *} \leq \delta_{1}, \quad \forall h, \\
\lambda_{1 h}^{*} \geq 0, \lambda_{2 h}^{*} \geq 0, \quad \forall h, \\
\lambda_{1 h}^{*}\left(x_{1}^{h *}-\delta_{1}\right)=0, \lambda_{2 h}^{*} x_{1}^{h *}, \quad \forall h, \\
x_{1}^{h *}+x_{0}^{h}-\mu+\lambda_{1 h}^{*}-\lambda_{2 h}^{*}+v^{*}=0, \quad \forall h .
\end{gathered}
$$

To satisfy the slackness condition of (A6), there are four possible cases:

(i) $\quad \lambda_{1 h}^{*}>0$, and $\lambda_{2 h}^{*}>0$ : No feasible solution exists.

(ii) $\quad \lambda_{1 h}^{*}=0$, and $\lambda_{2 h}^{*}>0$ : From (A7) and (A8), we have $x_{1}^{h *}=0$, and $x_{0}^{h}-\mu-\lambda_{2 h}^{*}+v^{*}=0$. From (A6), the latter equation is changed into $x_{0}^{h}>\mu-v^{*}$. Therefore, the condition to be in this case is $\mathcal{T}_{\text {min }}=\left\{h \in \mathcal{T}_{i} \mid x_{0}^{h}>\mu-v^{*}\right\}$, where $\mathcal{T}_{\text {min }}$ denotes the set of time intervals that are in this case.

(iii) $\quad \lambda_{1 h}^{*}>0$, and $\lambda_{2 h}^{*}=0$ : From (A7) and (A8), $x_{1}^{h *}=\delta_{1}$, and $\delta_{1}+x_{0}^{h}-\mu+\lambda_{1 h}^{*}+v^{*}=0$. Due to the slack variable, we have $x_{0}^{h}<\mu-\delta_{1}-v^{*}$. The set of time intervals for this case is $\mathcal{T}_{\text {max }}=\left\{h \in \mathcal{T}_{i} \mid x_{0}^{h}<\mu-\delta_{1}-v^{*}\right\}$. 
(iv) $\quad \lambda_{1 h}^{*}=0$, and $\lambda_{2 h}^{*}=0$ : Condition (A7) is satisfied, so we have $0 \leq x_{1}^{h *} \leq \delta_{1}$ and $x_{1}^{h *}+x_{0}^{h}-$ $\mu+v^{*}=0$. Therefore, $x_{1}^{h *}=\mu-x_{0}^{h}-v^{*}$. Let $\mathcal{T}_{\text {int }}$ denotes the set of time intervals that are in this case. Since this is the last case, it is defined as $\mathcal{T}_{\text {int }}=\mathcal{T}_{i} \backslash\left(\mathcal{T}_{\min } \cup \mathcal{T}_{\text {max }}\right)$. That is, $\mathcal{T}_{\text {int }}=\left\{h \in \mathcal{T}_{i} \mid \mu-\delta_{1}-v^{*} \leq x_{0}^{h} \leq \mu-v^{*}\right\}$.

Finally, we have

$$
x_{1}^{h *}= \begin{cases}0, & \text { if } h \in \mathcal{T}_{\text {min }} \\ \mu-x_{0}^{h}-v^{*}, & \text { if } h \in \mathcal{T}_{\text {int }} \\ \delta_{1}, & \text { if } h \in \mathcal{T}_{\text {max }} .\end{cases}
$$

From (A5), $v^{*}$ can be obtained as

$$
v^{*}=\frac{1}{\left|\mathcal{T}_{\text {int }}\right|}\left(\sum_{h \in \mathcal{T}_{\text {int }}}\left(\mu-x_{0}^{h}\right)+\left|\mathcal{T}_{\max }\right| \delta_{1}-\frac{E_{1}}{\mu_{c}}\right) .
$$

This solution shows an intuition that the EV consumes zero or maximum rate when the base load is higher or lower than a certain threshold, respectively. When the base load is in between the two thresholds, it consumes electricity between 0 and $\delta_{i}$ that is inversely proportional to the base load.

For the cases of more than one $\mathrm{EV}$, the problem $\left(\mathrm{C}^{\prime}\right)$ is still the convex optimization problem, though its closed form solution is a bit more complex. We can solve them by using the CVX package. CVX is a Matlab-based modeling system for convex optimization [37].

\section{References}

1. UNFCCC. Adoption of the Paris Agreement. Report No. FCCC/CP/2015/L.9/Rev.1 2015. Available online: http:/ / unfccc.int/resource/docs/2015/cop21/eng/109r01.pdf (accessed on 23 September 2017).

2. Trigg, T.; Telleen, P. Global EV Outlook—Understanding the Electric Vehicle Landscape to 2020; International Energy Agency: Paris, France, 2013.

3. Clement-Nyns, K.; Haesen, E.; Driesen, J. The Impact of Charging Plug-In Hybrid Electric Vehicles on a Residential Distribution Grid. IEEE Trans. Power Syst. 2010, 25, 371-380. [CrossRef]

4. Kim, J.-H.; Kim, C.-H. Smart EVs Charging Scheme for Load Leveling Considering ToU Price and Actual Data. J. Electr. Eng. Technol. 2017, 12, 1-10. [CrossRef]

5. Deilami, S.; Masoum, A.; Moses, P.; Masoum, M. Real-Time Coordination of Plug-in Electric Vehicle Charging in Smart Grids to Minimize Power Losses and Improve Voltage Profile. IEEE Trans. Smart Grid 2011, 2, 456-467. [CrossRef]

6. Sharma, I.; Canizares, C.; Bhattacharya, K. Smart Charging of PEVs Penetrating into Residential Distribution Systems. IEEE Trans. Smart Grid 2014, 5, 1196-1209. [CrossRef]

7. Tang, W.; Zhang, Y. A Model Predictive Control Approach for Low-Complexity Electric Vehicle Charging Scheduling: Optimality and Scalability. IEEE Trans. Power Syst. 2017, 32, 1050-1063. [CrossRef]

8. Hu, J.; You, S.; Lind, M.; Østergaard, J. Coordinated Charging of Electric Vehicles for Congestion Prevention in the Distribution Grid. IEEE Trans. Smart Grid 2014, 5, 703-711. [CrossRef]

9. Yoon, S.-G.; Choi, Y.-J.; Park, J.-K.; Bahk, S. Stackelberg Game based Demand Response for at-Home Electric Vehicle Charging. IEEE Trans. Veh. Technol. 2016, 65, 4172-4184. [CrossRef]

10. Gan, L.; Topcu, U.; Low, S. Optimal Decentralized Protocol for Electric Vehicle Charging. IEEE Trans. Power Syst. 2013, 28, 940-951. [CrossRef]

11. Sun, B.; Huang, Z.; Tan, X.; Tsang, D. Optimal Scheduling for Electric Vehicle Charging with Discrete Charging Levels in Distribution Grid. IEEE Trans. Smart Grid 2016. [CrossRef]

12. Venkataramanan, G.; Marnay, C. A Larger Role for Microgrids. IEEE Power Energy Mag. 2008, 6, 78-82. [CrossRef]

13. Kwasinski, A.; Krishnamurthy, V.; Song, J.; Sharma, R. Availability Evaluation of Micro-Grids for Resistant Power Supply during Natural Disasters. IEEE Trans. Smart Grid 2012, 3, 2007-2018. [CrossRef]

14. Wu, T.; Yang, Q.; Bao, Z.; Yan, W. Coordinated Energy Dispatching in Microgrid with Wind Power Generation and Plug-in Electric Vehicles. IEEE Trans. Smart Grid 2013, 4, 1453-1463. [CrossRef] 
15. Jian, L.; Xue, H.; Xu, G.; Zhu, X.; Zhao, D.; Shao, Z. Regulated Charging of Plug-in Hybrid Electric Vehicles for Minimizing Load Variance in Household Smart Microgrid. IEEE Trans. Ind. Electron. 2013, 60, 3218-3226. [CrossRef]

16. Hafez, O.; Bhattacharya, K. Optimal Planning and Design of a Renewable Energy Based Supply System for Microgrids. Renew. Energy 2012, 45, 7-15. [CrossRef]

17. Stadler, M.; Marnay, C.; DeForest, N.; Eto, J.; Cardoso, G.; Klapp, D.; Lai, J. Web-Based Economic and Environmental Optimization of Microgrids. In Proceedings of the IEEE Innovative Smart Grid Technologies (ISGT), Washington, DC, USA, 16-20 January 2012.

18. Khodaei, A.; Bahramirad, S.; Shahidehpour, M. Microgrid Planning under Uncertainty. IEEE Trans. Power Syst. 2015, 30, 2417-2425. [CrossRef]

19. Khodaei, A. Provisional Microgrid Planning. IEEE Trans. Smart Grid 2017, 8, 1096-1104. [CrossRef]

20. Narayan, A.; Ponnambalam, K. Risk-Averse Stochastic Programming Approach for Microgrid Planning under Uncertainty. Renew. Energy 2017, 101, 399-408. [CrossRef]

21. Richardson, P.; Flynn, D.; Keane, A. Optimal Charging of Electric Vehicles in Low-Voltage Distribution Systems. IEEE Trans. Power Syst. 2012, 27, 268-279. [CrossRef]

22. Electric Vehicle (EV) Rate Plans, PG\&E. Available online: https:/ /www.pge.com/en_US/residential/rate-plans / rate-plan-options/electric-vehicle-base-plan/electric-vehicle-base-plan.page (accessed on 22 August 2017).

23. Electric Rates Table, KEPCO. Available online: http://cyber.kepco.co.kr/ckepco/front/jsp/CY/E/E/ CYEEHP00202.jsp (accessed on 22 August 2017).

24. Acha, S.; Green, T.; Shah, N. Optimal Charging Strategies of Electric Vehicles in the UK Power Market. In Proceedings of the IEEE Innovative Smart Grid Technologies (ISGT), Anaheim, CA, USA, 17-19 January 2011.

25. HOMER Energy. Available online: http://www.homerenergy.com/ (accessed on 27 July 2017).

26. Net Present Value. Wikipedia. Available online: https://en.wikipedia.org/wiki/Net_present_value (accessed on 27 July 2017).

27. Lambert, T.; Gilman, P.; Lilienthal, P. Micropower System Modeling with HOMER. In Integration of Alternative Sources of Energy; Farret, F., Simões, M., Eds.; John Wiley \& Sons: Hoboken, NJ, USA, 2006.

28. South Australian Government, Data Collection of Diesel Generators in South Australia, September 2013. Available online: http:/ / www.renewablessa.sa.gov.au/investor-information/diesel-generators-directory (accessed on 27 July 2017).

29. Korea Energy Agency, Electrical Consumption Pattern Analysis by using the Customer Load Curve, March 2007. Available online: http:/ /www.kemco.or.kr/web/kem_home_new/info/data/open/kem_view. asp?sch_key=\&sch_value=\&sch_cat=\&c=306\&q=12847 (accessed on 27 July 2017).

30. NASA Surface Meteorology and Solar Energy Website. Available online: https:/ / eosweb.larc.nasa.gov/sse/ (accessed on 27 July 2017).

31. Diesel Prices. Available online: http:/ / www.globalpetrolprices.com/diesel_prices/ (accessed on 27 July 2017).

32. Nissan Leaf. Available online: https:/ / www.nissanusa.com/electric-hybrid-cars (accessed on 27 July 2017).

33. Summary of Travel Trends: 2009 National Household Travel Survey. Available online: http://nhts.ornl.gov/ (accessed on 27 July 2017).

34. Ortega-Vazquez, M.; Bouffard, F.; Silva, V. Electric Vehicle Aggregator/System Operator Coordination for Charging Scheduling and Services Procurement. IEEE Trans. Power Syst. 2013, 28, 1806-1815. [CrossRef]

35. Korea Meteorology Administration Website. Available online: http:/ / www.kma.go.kr/ (accessed on 27 July 2017).

36. Boyd, S.; Vandenberghe, L. Convex Optimization; Cambridge University Press: Cambridge, UK, 2004.

37. Grant, M.; Boyd, S. CVX: Matlab Software for Disciplined Convex Programming, Version 1.22. Available online: http:/ / cvxr.com/cvx (accessed on 27 July 2017).

(C) 2017 by the authors. Licensee MDPI, Basel, Switzerland. This article is an open access article distributed under the terms and conditions of the Creative Commons Attribution (CC BY) license (http:/ / creativecommons.org/licenses/by/4.0/). 